\title{
Age estimation for children and young adults by volumetric analysis of upper anterior teeth using cone-beam computed tomography data
}

\author{
Z. Yang1', L. Fan², K. Kwon'1, J. Pan'1, C. Shen'1, J. Tao ${ }^{3}$, F. Ji \\ 'Department of Orthodontics, Shanghai Ninth People's Hospital, College of Stomatology, Shanghai Jiao Tong University \\ School of Medicine, National Clinical Research Centre for Oral Diseases, Shanghai Key Laboratory of Stomatology and \\ Shanghai Research Institute of Stomatology, Shanghai, China \\ 2Department of Radiology, Shanghai Ninth People's Hospital, College of Stomatology, Shanghai Jiao Tong University \\ School of Medicine, National Clinical Research Centre for Oral Diseases, Shanghai Key Laboratory of Stomatology and \\ Shanghai Research Institute of Stomatology, Shanghai, China \\ ${ }^{3}$ Department of General Dentistry, Shanghai Ninth People's Hospital, College of Stomatology, Shanghai Jiao Tong \\ University School of Medicine, National Clinical Research Centre for Oral Diseases, Shanghai Key Laboratory of \\ Stomatology and Shanghai Research Institute of Stomatology, Shanghai, China
}

[Received: 11 December 2019; Accepted: 2 January 2020]

\begin{abstract}
Background: The aim of this study was to investigate the association between chronological age and the pulp/tooth volume ratio (PTR) of specific teeth using cone-beam computed tomography (CBCT) enhanced with Materialise-Mimics Research software 21.0 in children and young adult population from Eastern China. Materials and methods: CBCT scans of 230 patients (119 males, 111 females), aged 8.18-19.92 years were analysed by two well-trained examiners in this retrospective study. The intraclass correlation coefficient value was calculated to test the intra- and inter-examiner agreement. The volumetric analysis of the pulp and calcified tissues was performed on the maxillary left central incisors and canines. The correlation and regression analyses were then performed.

Results: The Pearson correlation analysis showed a strong coefficient of correlation ( $r$ ) for maxillary left canines (-0.81 for girls and -0.88 for boys) as compared to central incisors (-0.63 for girls and -0.70 for boys). Regarding performance, the canine model was more powerful than the central incisor model. The derived regression equation from maxillary left canines had high coefficients of determination (Age $=21.979-105.42 \times P T R, R^{2}=0.69$ ).

Conclusions: Our study proved that the PTR value of canines had a negative correlation relationship with a subadult's chronological age and volumetric analysis of CBCT scans using the software may become an efficient method to estimate the chronological age of children and young adults. (Folia Morphol 2020; 79, 4: 851-859)
\end{abstract}

Key words: dental age estimation, Mimics Software, Chinese children, cone-beam computed tomography, volumetric analysis

\footnotetext{
Address for correspondence: Dr. F. Ji, Department of Orthodontics, Shanghai Ninth People's Hospital, College of Stomatology, Shanghai Jiao Tong University School of Medicine, National Clinical Research Centre for Oral Diseases, Shanghai Key Laboratory of Stomatology and Shanghai Research Institute of Stomatology, Shanghai, China, e-mail: smilefang98@163.com; Dr. J. Tao, Department of General Dentistry, Shanghai Ninth People's Hospital, College of Stomatology, Shanghai Jiao Tong University School of Medicine, National Clinical Research Centre for Oral Diseases, Shanghai Key Laboratory of Stomatology and Shanghai Research Institute of Stomatology, Shanghai, China, e-mail: doctor_taojiang@126.com
} 


\section{INTRODUCTION}

Age estimation has vital importance in civil and criminal proceedings and clinic work and was generally utilised in identity verification of human remains, adjudication of criminal responsibility and determination of clinical treatment plans $[15,18]$. Dental age is an efficient method to estimate the chronological age of humans. The advantages of dental age assessment over other methods are its simplicity and less time consumption. In many circumstances, tooth tends to be the only remained tissue of human remains we could utilize to estimate the chronological age quickly, since dental enamel is the hardest tissue in the human body with exceptional resistance to physical and chemical forces and tooth development is regulated under strict genetic control $[7,13,16,21]$.

Systematic research continued for several decades [23], and many methods were proposed to estimate the dental age of children. The most widely used methods included the qualitative methods that relied on the manual assessment of the shape of the developing tooth by the naked eye, such as Demirjian's method [9] and Willems's method [27], and qualitative method, such as Cameriere's method [6]. As most of the techniques were based on samples from a foreign population, many scientists attempted to test their applicability to the Chinese population. Ye et al. [28] selected 941 orthopantomograms of 7-14-year-old children from Eastern China and found that the Willems's method was more accurate in estimating the dental age than the Demirjian's method [28]. For the 11-18-year-olds from Eastern China, the Demirjian's method was more reliable compared to the Willems's method according to Wang et al. [26]. They conclude that the Demirjian's method was unsatisfactory for 11-18-year-olds from Eastern China [26]. Evidently, several researches showed different, even contradictory results. This could be because manual observation of the shape of the tooth was readily influenced by the personal interpretation.

The shape of a tooth changes throughout life, especially during its developing stage. The apical foramen becomes smaller in diameter; the crown develops with the formation of enamel. Under this circumstance, Cameriere et al. [6] estimated the dental age of children quantitatively by the extent of the apical foramen's opening (the Cameriere's method). They assessed the maturity of teeth by recording the number of teeth with completely closed apical foramen, the distance between the inner point of the open apical foramen and the length of the teeth [6].

In fact, even after completion of tooth development, the tooth's morphology continues to show age-related changes. With the deposition of secondary dentin along the inner walls of the pulp chamber, the pulp/tooth ratio (PTR) reduces continually $[4,17]$. Hence, there is an obvious inverse correlation relationship between chronological age and the PTR value and many studies started using this parameter to estimate the chronological age of adults [1-3, 8, 10-12, 14, 20]. Cameriere et al. [6] collected orthopantomograms of 100 Italian, White, Caucasian subjects, between 18 to 72 years of age. PTR, tooth length, pulp/tooth length ratio, pulp/tooth area and pulp/root width ratios of maxillary right canines at three different levels were computed and the ratio between pulp and tooth area correlated best with age $\left(r^{2}=0.85\right)[5]$.

However, all the methods mentioned above are based on the two-dimensional (2D)-panoramic radiographs and there is always some detailed information of teeth is lost.

With the development of medical technology, cone-beam computed tomography (CBCT) has become a valuable tool in dental clinics. This is due to its lower radiation dose and cost-efficacy as compared to traditional medical computed tomography. Moreover, it has greater metric accuracy with isotropic voxel resolutions [19]. The $C B C T$ image analysis can be enhanced by Materialise-Mimics Research software 21.0. Using its segment function, we can now generate the three-dimensional (3D) model of the scanned structure and acquire its superficial area and volume [26], then the pulp/tooth ratio could be calculated.

In addition, many previous studies focusing on the relationship between chronological age and the pulp/tooth volume ratio included samples of all age groups from young to old, not a specific age group $[2,3,12,29]$. Thus far, no study to date has used PTR value to estimate dental age of children.

So, our interest was aroused that if the PTR value derived from $C B C T$ data could be utilized to estimate the chronological age of children.

As the canines are the most stable teeth in situ and subject less wearing [8, 24], the central incisors had been studied in many researches which are convenient to be compared and both teeth types are mono-radicular. So, we selected the maxillary left canines and the central incisors to examine the efficacy 
of the CBCT technique for dental age estimation of children from Eastern China.

\section{MATERIALS AND METHODS}

\section{Ethics issues}

The research was approved by the independent ethics committee of the Shanghai Ninth People's Hospital affiliated with Shanghai Jiao Tong University, School of Medicine (No: 2017-284-T212).

\section{Sample and eligibility criteria}

Cone-beam computed tomography data were randomly selected from 8-19-year-old patients at Shanghai Ninth People's Hospital between January 2015 and May 2019. All CBCT data was obtained for diagnosis and therapeutic purposes, and no subjects suffered additional radiation exposure.

The inclusion criteria were Han Chinese between 8 to 19 years of age and clear CBCT image.

The exclusion criteria were severe jaw defects or deformities, missing maxillary central incisors or canines, severe caries or defects on maxillary central incisors or maxillary canines, and severe systemic disease.

\section{Data collection}

All the $C B C T$ data were acquired using the same Planmeca ProMax 3D Max machine. The pixel size was 200 , and the slice thickness was $0.2 \mathrm{~mm}$. The selected scans had an exposure parameter of $96 \mathrm{kV}, 8.0 \mathrm{~mA}$, and the exposure time was approximately $12 \mathrm{~s}$. The dose area product was $1289 \mathrm{mGy} \times \mathrm{cm}^{2}$.

A total of 230 children (aged 8-19 years) were included in our study. The samples were divided into 12 groups, according to age. Ten boys and ten girls were included in each group to ensure balance in data component distribution. For each subject of our study, we collected the following details: age, sex, date of birth, date of CBCT scan, and the DICOM information of the CBCT data. Our study included 228 maxillary left incisors and 229 maxillary left canines. The specific composition of our samples is shown in Tables 1 and 2.

\section{Measurements}

For the training group, 10 samples were randomly selected to calculate the intraclass correlation coefficient (ICC). The PTR values of these 10 maxillary left canines were measured separately at the same time by two examiners to test the inter-examiner agreement
Table 1. The distribution of samples according to age and sex

\begin{tabular}{lccc}
\hline Age & Boys & Girls & Total \\
\hline 8 & 10 & 8 & 18 \\
9 & 8 & 8 & 16 \\
10 & 10 & 10 & 20 \\
11 & 12 & 7 & 19 \\
12 & 8 & 10 & 18 \\
13 & 11 & 10 & 21 \\
14 & 9 & 8 & 17 \\
15 & 10 & 9 & 19 \\
16 & 12 & 9 & 21 \\
17 & 9 & 10 & 19 \\
18 & 9 & 11 & 20 \\
19 & 11 & 11 & 22 \\
Total & 119 & 111 & 230 \\
\hline
\end{tabular}

Table 2. The distribution of examined teeth according to age group and sex

\begin{tabular}{lcccccc}
\hline \multirow{2}{*}{ Age [years] } & \multicolumn{2}{c}{ Tooth 21 } & & \multicolumn{2}{c}{ Tooth 23 } & Total \\
\cline { 2 - 3 } \cline { 7 - 7 } & Boy & Girl & & Boy & Girl & \\
\hline 8 & 10 & 8 & & 10 & 8 & 36 \\
9 & 8 & 8 & & 8 & 8 & 32 \\
10 & 10 & 10 & & 10 & 10 & 40 \\
11 & 12 & 7 & & 12 & 7 & 38 \\
12 & 8 & 10 & & 8 & 10 & 36 \\
13 & 11 & 10 & & 11 & 10 & 42 \\
14 & 9 & 8 & & 9 & 8 & 34 \\
15 & 10 & 9 & & 10 & 9 & 38 \\
16 & 12 & 9 & & 12 & 9 & 42 \\
17 & 7 & 10 & & 9 & 9 & 35 \\
18 & 9 & 11 & 9 & 11 & 40 \\
19 & 11 & 11 & & 11 & 11 & 44 \\
Total & 117 & 111 & & 119 & 110 & 457 \\
\hline
\end{tabular}

and remeasured by one examiner with 2 weeks' interval to test the intra-examiner agreement. Then we measured the PTR value of the maxillary left central incisor and canines for each sample. If the maxillary left central incisor or canine was missing or could not be analysed due to severe caries or defects, its counterpart on the other side was analysed.

Firstly, the DICOM files, which contained 651 independent subfiles, were imported into the software to construct the 3D models. The number of slices was sufficient to obtain the precise volume of the hard tissue and pulp for each tooth. The more slices 


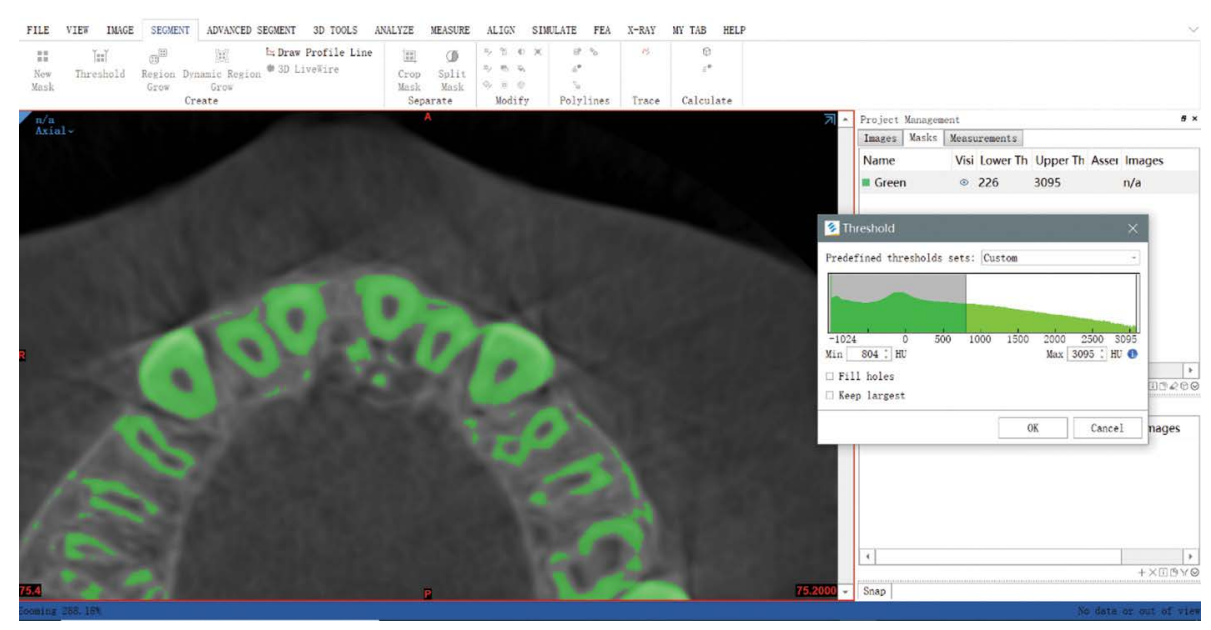

Figure 1. Threshold range selecting process.

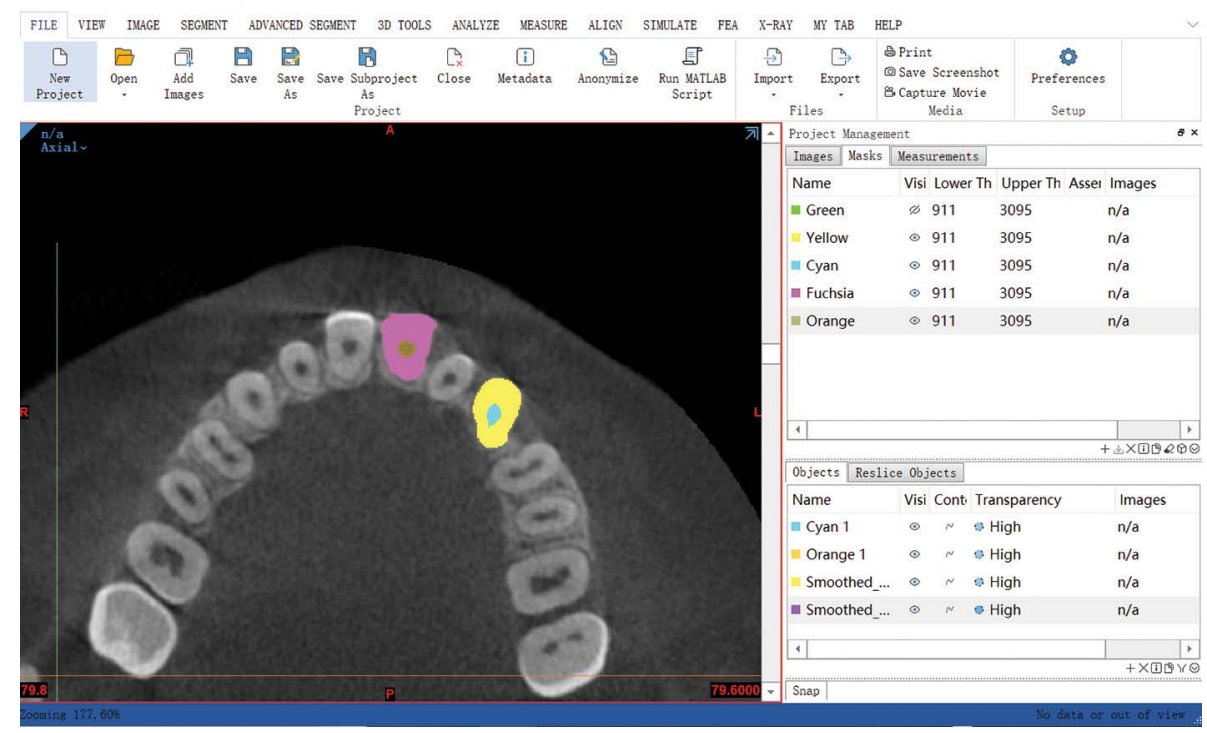

Figure 2. Multiple slice editing process (axial plane).

one CBCT sample had, the smaller slice thickness it would have, and we could precisely define the tooth structure and obtain more accurate data.

Secondly, the appropriate threshold range was set to highlight the hard tissue of the tooth (Fig. 1). Once determined, it would not be altered during the entire process of 3D model construction. The false-positive highlight area around the hard tissue of the tooth, that generated due to the short width of periodontal attachment, the similar density of the bone around the tooth cementum, or other mixed pixels, was manually removed from the highlight area using multiple slice editing function in the axial plane to ensure the accuracy of the volume data obtained. (Figs. 2, 3) The region growing function was used to separate the tooth from other tissues and generated a new mask with a different colour.

Thirdly, we separated the hard tissue from the pulp cavity of the tooth by cavity fill function. The pulp cavity area of the tooth was surrounded by the highlight area of the hard tissue, which could be filled in using another highlight colour and a new mask representing the pulp cavity was then constructed. For those teeth whose apical foramen were still open, we sealed the mask in the apical foramen of the tooth using the adding functions of the multiple slice editing functions to turn the pulp cavity from an open space into a closed space. Since we analysed two teeth for each sample, the hard tissue area and the pulp cavity area of each tooth needed two highlight 


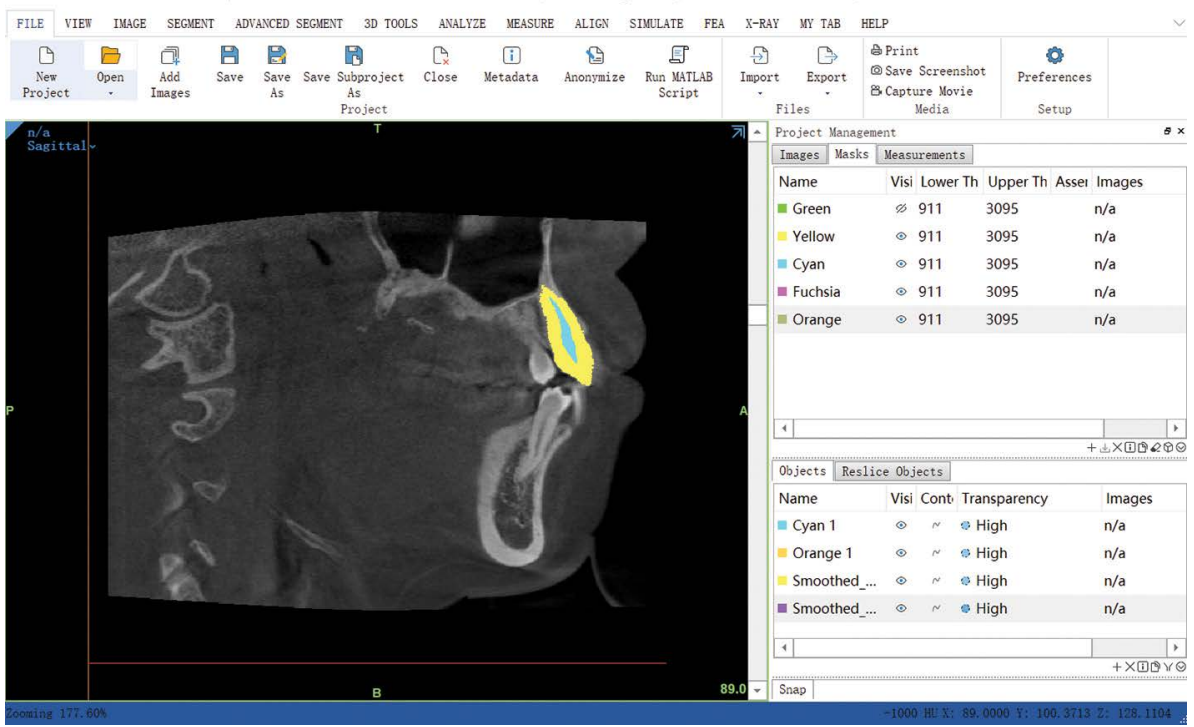

Figure 3. Multiple slice editing process (sagittal plane).

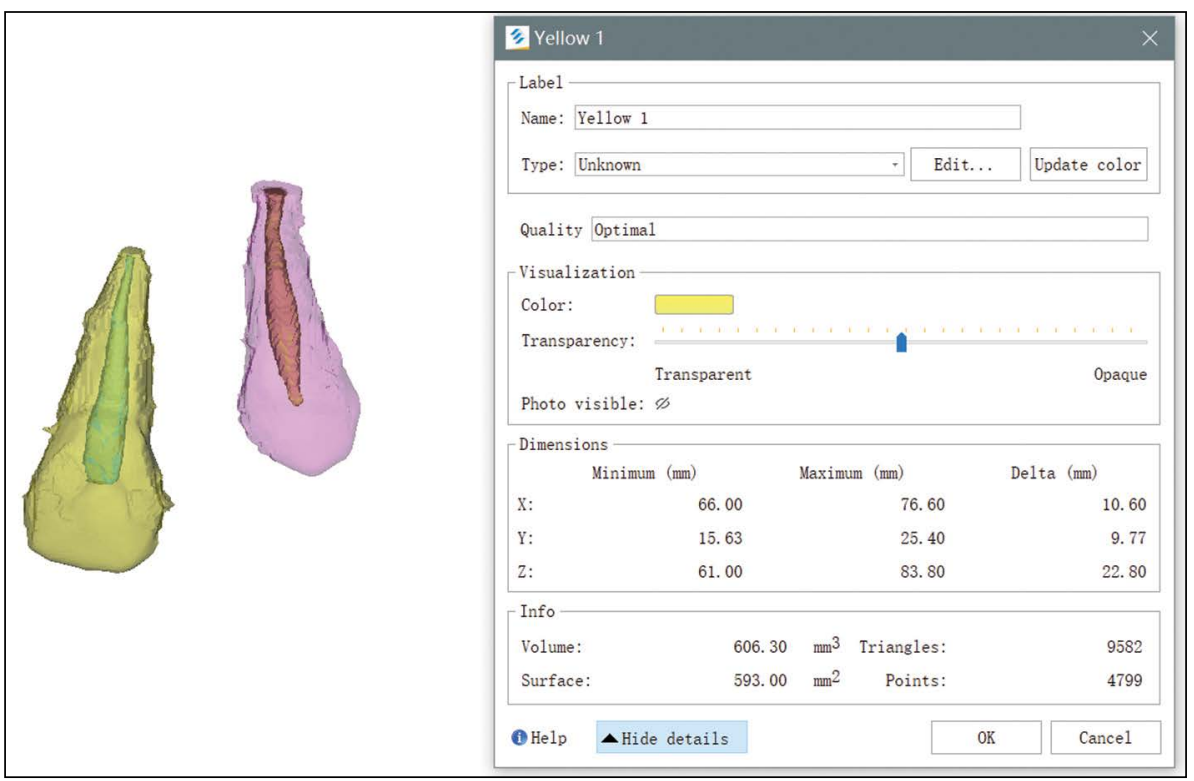

Figure 4. Construction of the three-dimensional model and the volume measuring process.

colours, respectively. In that way, five different masks with five different colours would be created for each sample in the end.

Lastly, the four masks which represented the hard tissue and the pulp cavity of the maxillary central incisor and the maxillary canine respectively were transformed to 3D models, and the volume $\left(\mathrm{mm}^{3}\right)$ of each part could be calculated by the properties function (Fig. 4). In the end, the pulp/tooth value of each tooth could be obtained.

\section{Statistical analysis}

All statistical values were analysed using Microsoft Excel 2019 and the SAS V8 and the significance level for this study was set to $5 \%$. The following morphological variables were recorded for each sample's maxillary left central incisor and canine: $\mathrm{pv}=$ the volume of pulp cavity, $t v=$ the volume of the entire tooth, and PTR = pulp/tooth volume ratio. The Pearson correlation coefficient between the PTR value and the chronological age was calculated. 
Fisher $\mathrm{Z}$ test analysis was performed to test the statistical difference in the correlation coefficient values between the central incisor model and canine model.

Linear regression was performed using chronological age as the dependent variable and PTR value as the independent variable. The standard errors of estimate were then calculated to analyse the reliability of different regression equations.

\section{RESULTS}

The main error came from two points: 1) The threshold range selected for the segment process; 2) The multiple slices editing operation to separate the tooth from the surrounding tissues.

To eliminate the error, we calculated the inter-examiner $(\mathrm{ICC}=0.989,95 \% \mathrm{CI}(\mathrm{L})=0.955,95 \% \mathrm{Cl}(\mathrm{U})$ $=0.997)$ and intra-examiner $(\mathrm{ICC}=0.973,95 \% \mathrm{CI}(\mathrm{L})$ $=0.973,95 \% \mathrm{Cl}(\mathrm{U})=0.993)$ agreement, which showed an excellent reproducibility of this measurement.

The sample comprised of 119 boys and 111 girls. The mean, standard deviation, minimum and maximum of the ages of all boys and girls was shown in Table 3. The mean, standard deviation, minimum and maximum of the PTR values of all the analysed teeth were shown in Table 4.

There was a significant correlation between chronological ages and PTR of central incisors or canines for both boys and girls $(p<0.01)$. The correlation coefficients were -0.70 (boy group), -0.63 (girl group) and -0.67 (global samples) for central incisors, and -0.88 (boy group), -0.81 (girl group) and -0.83 (global samples) for canines, respectively. Fisher $Z$ test showed a significant difference in the correlation coefficient values between maxillary central left incisor and maxillary left canine. However, there was no significant difference in the correlation relationship between genders for both tooth positions (Tables 5, 6). In this circumstance, we combine the data and two regression equations were derived from the acquired data. The higher $R^{2}=0.69$ was found from the regression equation derived from the maxillary canines which meant that the regression equation could explain $69 \%$ of the total variance of age. The standard error of estimate (SEE) of two estimation models was calculated. The results showed that the maxillary left canine model had the smaller SEE than the central incisor model (Table 7).

The scatter plot of chronological age-pulp/tooth ratio for all three models is shown in Figures 5 and 6 .
Table 3. The mean, standard deviation (SD), minimum (Min) and maximum (Max) of the ages of all boys and girls

\begin{tabular}{lcccc}
\hline Sex & Mean & SD & Min & Max \\
\hline Boy & 13.99 & 3.45 & 8.19 & 19.92 \\
Girl & 14.29 & 3.47 & 8.48 & 19.92 \\
\hline
\end{tabular}

Table 4. The mean, standard deviation (SD), minimum (Min) and maximum (Max) of the pulp/tooth volume ratio values of all the analysed teeth

\begin{tabular}{|c|c|c|c|c|c|c|c|c|}
\hline \multirow[t]{2}{*}{ Sex } & \multicolumn{2}{|c|}{ Mean } & \multicolumn{2}{|c|}{ SD } & \multicolumn{2}{|c|}{ Min } & \multicolumn{2}{|c|}{ Max } \\
\hline & 21 & 23 & 21 & 23 & 21 & 23 & 21 & 23 \\
\hline Boy & 0.053 & 0.080 & 0.024 & 0.030 & 0.016 & 0.032 & 0.137 & 0.148 \\
\hline Girl & 0.049 & 0.069 & 0.020 & 0.022 & 0.006 & 0.022 & 0.105 & 0.124 \\
\hline Total & 0.051 & 0.075 & 0.022 & 0.027 & 0.006 & 0.022 & 0.137 & 0.148 \\
\hline
\end{tabular}

Table 5. Fishers $Z$ test to test the significant difference in the correlation coefficient between maxillary left central incisor and canine

\begin{tabular}{cccccc}
\hline \multicolumn{2}{c}{ Correlation coefficient } & & \multicolumn{2}{c}{ Fishers Z test } \\
\cline { 1 - 2 } \cline { 5 - 6 } 21 & $\mathbf{2 3}$ & & Z value & P value \\
\hline-0.665 & -0.833 & & 12.07 & $<0.05$ \\
\hline
\end{tabular}

Table 6. Fishers $Z$ test to test the significant difference in the correlation coefficient between genders for both tooth position

\begin{tabular}{lcclcc}
\hline Model & \multicolumn{2}{c}{ Correlation coefficient } & & \multicolumn{2}{c}{ Fishers Z test } \\
\cline { 2 - 3 } \cline { 5 - 6 } type & Boy & Girl & & Z value & P value \\
\hline 21 & -0.70 & -0.63 & & 0.94 & $>0.05$ \\
23 & -0.88 & -0.81 & & 1.72 & $>0.05$ \\
\hline
\end{tabular}

Table 7. The comparison of the regression equation between the maxillary left central incisor group and canine group

\begin{tabular}{lcccc}
\hline Tooth & $\mathbf{N}$ & $\mathbf{R}^{2}$ & Regression equation & SEE \\
\hline 21 & 228 & 0.44 & Age $=19.35-102.73 \times$ PTRI & 2.58 \\
23 & 229 & 0.69 & Age $=21.979-105.42 \times$ PTRC & 1.91
\end{tabular}

PTRC — the pulp/tooth ratio value of canine; PTRI — the pulp/tooth ratio value of central incisor; SEE - standard error of estimate

\section{DISCUSSION}

Several scientists have studied the correlation between age and decrease in pulp volume caused by the deposition of secondary dentin and applied this phenomenon to age estimation. As the tooth is a 3D structure, dental age could not be accurately assessed from 2D orthopantomograms, especially 


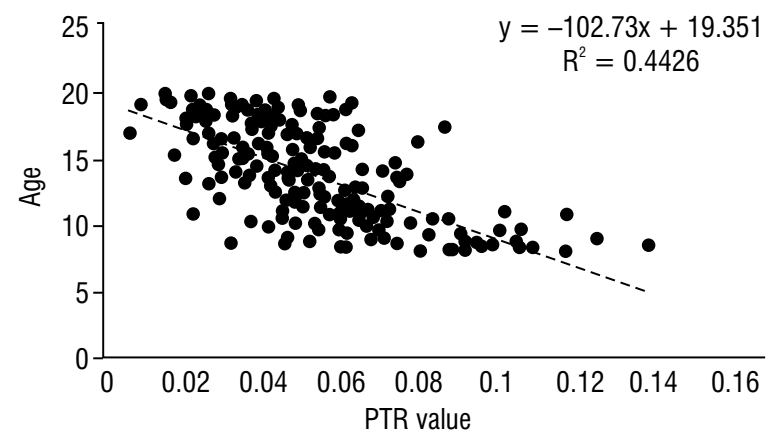

Figure 5. The relationship between the chronological age and the pulp/tooth ratio (PTR) value of the maxillary central incisor.

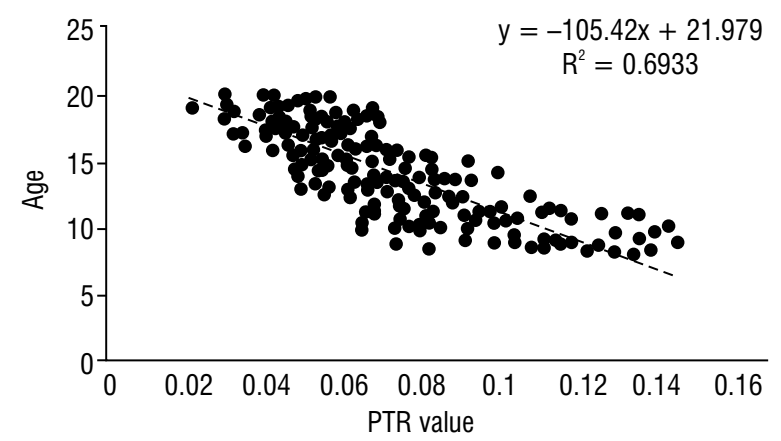

Figure 6. The relationship between the chronological age and the pulp/tooth ratio (PTR) value of the maxillary left canines.

when we could not get enough teeth from human remains $[11,20]$.

Many studies have proved that there exists no statistically significant difference between the right and left counterpart teeth in determining the efficacy of dental age estimation [3, 29]. Hence, we only analysed the teeth on the left side or the counterpart tooth in the contralateral quadrant, if the tooth on the left side was lost or malformed.

In our study, the correlation between the PTR and the chronological age in maxillary canines $(r=-0.833)$ was higher than that found in maxillary central incisors $(r=-0.665)$. The maxillary left canine estimation model, that was better than the central incisor estimation model, had a coefficient of determination of 0.77 for boys and 0.66 for girls. Gulsahi et al. [12] observed both maxillary central incisors and maxillary canines in one study, and the strongest correlation strength was found between the chronological age and PTR measured on maxillary central incisors $\left(r^{2}=0.532\right)$. Asif et al. [3] also calculated the PTR ratio of maxillary canines and central incisors using CBCT data and got the similar results. Some studies also analysed the maxillary central incisors or the maxillary canines using $\mathrm{CBCT}$ data but without calculating the PTR value. Asif et al. [2] analysed both the PTR and the pulp chamber/crown ratio and found that the second method had a higher $\mathrm{R}^{2}(0.775)$ than the PTR method. Ignoring the tooth position, Zhang et al. [29] found that the scope of $R^{2}$ by CBCT images for age estimation was about $0.21-0.70$.

In general, the strength of correlation in our study seems stronger than the previous studies. The reason may the decrease of PTR value was caused by different factors in subadults and adults. For subadults, the shape of tooth mainly changed with the deposition of enamel, dentin, and cementum and the closure of apical foramen. For adults, the shape of the tooth principally changed with the deposition of the secondary dentin. In that circumstance, it was reasonable to find different extents of correlation relationship between young people and people of all age.

Most studies showed a stronger correlation in the maxillary central incisor than the maxillary canines, which was contrary to our study. So, we may conclude that the PTR value of a central incisor was the better reference variance to estimate the age of adults comparing to a canine, while a canine is better when estimating the age of subadults. This could be because the age of apex formation for canines was 13-15 years, but for central incisors, it was around 10 years. Since the age range of our samples was 8-19 years, the variation of shape was more apparent and more sustainable in maxillary canines than the central incisors. Hence, it is quite reasonable to find that the association between PTR values and the chronological age in maxillary canines was stronger than the maxillary central incisors for children and young adults.

Our study didn't show a distinct sex dimorphism in age estimation. However, there are differences in the growth pattern between boys and girls. The growth spurt of girls precedes that of boys by approximately 2 years. Many methods for estimating chronological ages of children, such as the Demirjian's method and the Willems's method, showed significant sex dimorphism. However, most studies analysing the PTR values found no significant difference in the correlation coefficient values between genders for each type of investigated teeth $[3,12]$. This might be because all the subjects were adults and the dentin deposition pattern probably the same for males and females whose growth and development had ceased. For our study, the relatively small number of samples 
may be the cause. In fact, the $Z$ value $(Z=1.72)$ was quite close to 1.96 when we compared the $R$ values between genders. If we had collected more samples, the result may well be different.

In addition, the method we used to acquire the pulp and tooth volume was different from other studies, and the differences were shown in the modelling setup process after the CBCT data in DICOM files were imported into the software. In previous studies, new masks were created for the pulp cavity and the entire tooth after setting different grayscale threshold values for each of the investigated teeth [3]. In our opinion, multiple sets of grayscale values meant a higher probability of error. In our study, we set the grayscale value once to separate the hard tissue of the tooth and used the cavity filling function to define the pulp area. It was not only more accurate, but also less time-consuming. Most of the studies reported that the entire time of importing the DICOM files to the software, thresholding, and 3D reconstruction took around 2-3 hours per tooth $[3,22,25]$. Pinchi et al. [20] simplified dental volume measurement through a geometric approximation of the different parts of the tooth and spent less than 15 minutes per tooth. Through one-step thresholding and multiple slice editing, we could finish the $3 \mathrm{D}$ reconstruction process in about 20 minutes without geometric approximation.

We couldn't compare the efficiency of our new equation with Demirjian's or Williems's method since there wasn't an independent validation group, whereas it is very important. Since the $2 \mathrm{D}$ technique is less time-consuming, our method merits application only if it is more accurate than the Demirjian's or Williems's method. We decided to screen samples who took $\mathrm{CBCT}$ and panoramic radiographs on the same day to conduct the follow-up investigation.

\section{CONCLUSIONS}

We concluded that the maxillary left central incisors and left canines had a significant negative correlation relationship with the chronological age of children and young adults. The calculation of pulp-tooth ratio value using the data acquired from $\mathrm{CBCT}$ might be an effective method to estimate the chronological age of children and young adults. It is more reliable to estimate the chronological age by the PTR value of the maxillary left canines than left incisors and two regression equations were established respectively using the pulp-tooth value of the maxillary left canines and central incisors as the dependent variance. The accuracy of this estimation method is considerably high, whereas it is necessary to compare the accuracy of our new regression equation with other widely used methods using independent validation samples. Hence, there is a need to carry out further studies in the future.

\section{Acknowledgements}

This work was supported by grants from Interdisciplinary Programme of SJTU (YG2019ZDA07) and SJTU (BYH20180208; YH20180308).

\section{REFERENCES}

1. Andrade VM, Fontenele RC, de Souza ACb, et al. Age and sex estimation based on pulp cavity volume using cone beam computed tomography: development and validation of formulas in a Brazilian sample. Dentomaxillofac Radiol. 2019; 48(7): 20190053, doi: 10.1259/dmfr.20190053, indexed in Pubmed: 31322923.

2. Asif MK, Nambiar P, Mani SA, et al. Dental age estimation employing CBCT scans enhanced with Mimics software: Comparison of two different approaches using pulp/ /tooth volumetric analysis. J Forensic Leg Med. 2018; 54: 53-61, doi: 10.1016/j.jflm.2017.12.010, indexed in Pubmed: 29324319.

3. Asif MK, Nambiar P, Mani SA, et al. Dental age estimation in Malaysian adults based on volumetric analysis of pulp/ tooth ratio using CBCT data. Leg Med (Tokyo). 2019; 36: 50-58, doi: 10.1016/j.legalmed.2018.10.005, indexed in Pubmed: 30415192.

4. Bodecker CFA. consideration of some ofthe changes in the teeth from young to old age. Dental Cosmos. 1925; 67(6): 543-549.

5. Cameriere R, Ferrante L, Cingolani M. Variations in pulp/ /tooth area ratio as an indicator of age: a preliminary study. J Forensic Sci. 2004; 49(2): 317-319, indexed in Pubmed: 15027553.

6. Cameriere R, Ferrante L, Cingolani M. Age estimation in children by measurement of open apices in teeth. Int J Legal Med. 2006; 120(1): 49-52, doi: 10.1007/s00414005-0047-9, indexed in Pubmed: 16283352.

7. Chun KY, Choi Hh, Lee Jy. Comparison of mechanical property and role between enamel and dentin in the human teeth. J Dent Biomech. 2014; 5: 1758736014520809 , doi: 10.1177/1758736014520809, indexed in Pubmed: 24550998.

8. De Angelis $D$, Gaudio $D$, Guercini N, et al. Age estimation from canine volumes. Radiol Med. 2015; 120(8): 731-736, doi: 10.1007/s11547-015-0521-5, indexed in Pubmed: 25698302.

9. Demirjian A, Tanner HGM, Jain V, et al. A new system of dental age assessment. Hum Biol. 1973; 45(2): 211-227, indexed in Pubmed: 4714564.

10. Ge ZP, Yang P, Li G, et al. Age estimation based on pulp cavity/chamber volume of 13 types of tooth from cone beam computed tomography images. Int J Legal Med. 2016; 130(4): 1159-1167, doi: 10.1007/s00414-016-1384-6, indexed in Pubmed: 27221534. 
11. Ge Zp, Ma Rh, Li G, et al. Age estimation based on pulp chamber volume of first molars from cone-beam computed tomography images. Forensic Sci Int. 2015; 253: 133. e1-133.e7, doi: 10.1016/j.forsciint.2015.05.004, indexed in Pubmed: 26031807.

12. Gulsahi A, Kulah CK, Bakirarar B, et al. Age estimation based on pulp/tooth volume ratio measured on cone-beam CT images. Dentomaxillofac Radiol. 2018; 47(1): 20170239, doi: 10.1259/dmfr.20170239, indexed in Pubmed: 28991500.

13. Jelliffe EF, Jelliffe DB. Deciduous dental eruption, nutrition and age assessment. J Trop Pediatr Environ Child Health. 1973; 19(2): 193-248, indexed in Pubmed: 4492789.

14. Kazmi S, Mânica S, Revie G, et al. Age estimation using canine pulp volumes in adults: a CBCT image analysis. Int J Legal Med. 2019; 133(6): 1967-1976, doi: 10.1007/ s00414-019-02147-5, indexed in Pubmed: 31471652.

15. Kringsholm B, Jakobsen J, Sejrsen B, et al. Unidentified bodies/skulls found in Danish waters in the period 1992-1996. Forensic Sci Int. 2001; 123(2-3): 150-158, doi: 10.1016/s0379-0738(01)00533-3.

16. Laurencin D, Wong A, Chrzanowski W, et al. Probing the calcium and sodium local environment in bones and teeth using multinuclear solid state NMR and X-ray absorption spectroscopy. Phys Chem Chem Phys. 2010; 12(5): 1081-1091, doi: 10.1039/b915708e, indexed in Pubmed: 20094673.

17. Maples WR. An improved technique using dental histology for estimation of adult age. J Forensic Sci. 1978; 23(4): 764-770, indexed in Pubmed: 744998.

18. Panchbhai AS. Dental radiographic indicators, a key to age estimation. Dentomaxillofac Radiol. 2011; 40(4): 199-212, doi: 10.1259/dmfr/19478385, indexed in Pubmed: 21493876.

19. Patcas R, Markic G, Müller L, et al. Accuracy of linear intraoral measurements using cone beam CT and multidetector CT: a tale of two CTs. Dentomaxillofac Radiol. 2012; 41(8): 637-644, doi: 10.1259/dmfr/21152480, indexed in Pubmed: 22554987.

20. Pinchi V, Pradella F, Buti J, et al. A new age estimation procedure based on the 3D CBCT study of the pulp cavity and hard tissues of the teeth for forensic purposes: A pilot study. J Forensic Leg Med. 2015; 36: 150-157, doi: 10.1016/j. jflm.2015.09.015, indexed in Pubmed: 26458182.
21. Shekhawat KS, Chauhan A. Analysis of dental hard tissues exposed to high temperatures for forensic applications: An in vitro study. J Forensic Dent Sci. 2016; 8(2): 90-94, doi: 10.4103/0975-1475.186366, indexed in Pubmed: 27555725.

22. Star $H$, Thevissen $P$, Jacobs $R$, et al. Human dental age estimation by calculation of pulp-tooth volume ratios yielded on clinically acquired cone beam computed tomography images of monoradicular teeth. J Forensic Sci. 2011; 56 Suppl 1: S77-S82, doi: 10.1111/j.15564029.2010.01633.x, indexed in Pubmed: 21182523.

23. Stavrianos C, Mastagas D, Stavrianou I, et al. Dental age estimation of adults: A review of methods and principals. Res J Med Sci. 2008; 2: 258-268.

24. Tardivo D, Sastre J, Ruquet M, et al. Three-dimensional modeling of the various volumes of canines to determine age and sex: a preliminary study. J Forensic Sci. 2011; 56(3): 766-770, doi: 10.1111/j.1556-4029.2011.01720.x, indexed in Pubmed: 21361946.

25. Tardivo D, Sastre J, Catherine JH, et al. Age determination of adult individuals by three-dimensional modelling of canines. Int J Legal Med. 2014; 128(1): 161-169, doi: 10.1007/ s00414-013-0863-2, indexed in Pubmed: 23624552.

26. Wang J, Bai $X$, Wang $M$, et al. Applicability and accuracy of Demirjian and Willems methods in a population of Eastern Chinese subadults. Forensic Sci Int. 2018; 292: 90-96, doi: 10.1016/j.forsciint.2018.09.006, indexed in Pubmed: 30286341.

27. Willems G, Olmen AnV, Spiessens B, et al. Dental Age Estimation in Belgian Children: Demirjian's Technique Revisited. J Forensic Sci. 2001; 46(4), doi: 10.1520/jfs15064j.

28. Ye $X$, Jiang $F$, Sheng $X$, et al. Dental age assessment in 7-14-year-old Chinese children: comparison of Demirjian and Willems methods. Forensic Sci Int. 2014; 244: 36-41, doi: 10.1016/j.forsciint.2014.07.027, indexed in Pubmed: 25195126.

29. Zhang ZY, Yan CX, Min QM, et al. Age estimation using pulp/enamel volume ratio of impacted mandibular third molars measured on CBCT images in a northern Chinese population. Int J Legal Med. 2019; 133(6): 1925-1933, doi: 10.1007/s00414-019-02112-2, indexed in Pubmed: 31273446 . 